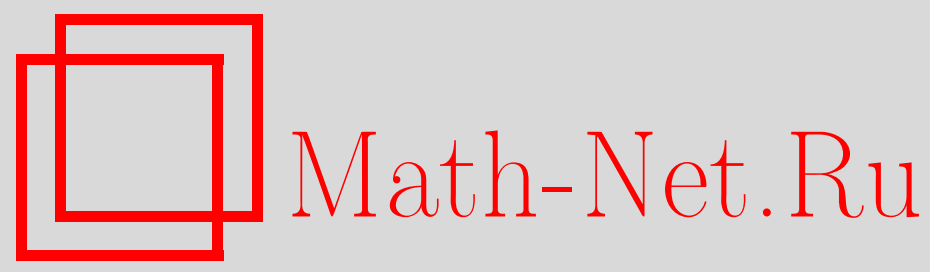

D. V. Denisenko, M. V. Nikitenkova, Оптимизация квантовых схем для $S$-боксов ГОСТ Р 34.12-2015 «Магма» без вспомогательных кубитов, Матем. вопр. криптогр., 2020, том 11, выпуск 2, 43-52

DOI: https://doi.org/10.4213/mvk320

Использование Общероссийского математического портала Math-Net.Ru подразумевает, что вы прочитали и согласны с пользовательским соглашением http://www . mathnet.ru/rus/agreement

Параметры загрузки:

IP : 52.6 .47 .48

26 апреля 2023 г., $17: 26: 13$

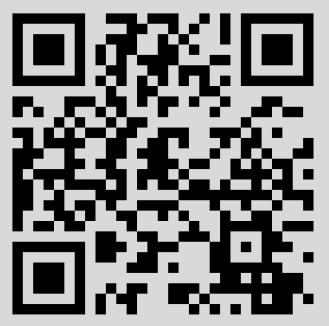




\title{
Optimization of S-boxes GOST R 34.12-2015 «Magma» quantum circuits without ancilla qubits
}

\author{
D. V. Denisenko, M. V. Nikitenkova
}

Bauman Moscow State Technical University (BMSTU), Moscow

Получено 05.XI.2019

Abstract. We study the ways to implement $S$-boxes by quantum circuits with a minimal number of logical qubits and logical quantum gates without using ancilla qubits. New quantum circuits that implement the $S$-boxes of the GOST R 34.12-2015 "Magma" on 4 logical qubits are constructed. It means that for substitutions $s \in S\left(V_{n}\right)$ with a large number of cycles there exist quantum circuits on $n$ logical qubits that implement the substitution $s$ with fewer logical quantum gates compared with substitutions $g \in S\left(V_{n}\right)$ with a small number of cycles.

Keywords: S-box, quantum circuit, resource estimates

Оптимизация квантовых схем для $S$-боксов ГОСТ Р 34.12-2015 «Магма» без вспомогательных кубитов

\section{Д. В. Денисенко, М. В. Никитенкова}

Московский государственный технический университет им.Н.Э.Баумана (МГТУ), Москва

Аннотация. Исследуются способы реализации $S$-боксов квантовыми схемами с минимальным количеством кубитов без использования вспомогательных кубитов. Получены новые квантовые схемы, реализующие $S$-боксы алгоритма GOST R 34.12-2015 «Магма» на 4 кубитах. Показано, что для подстановок $s \in S\left(V_{n}\right)$ с большим количеством циклов существуют квантовые схемы на $n$ кубитах, реализующие подстановку $s$ меньшим количеством квантовых вентилей, чем для подстановок $g \in S\left(V_{n}\right)$ с небольшим количеством циклов.

Ключевые слова: S-бокс, квантовые схемы, оценка квантовых ресурсов для реализации подстановок 


\section{Introduction}

The theory of quantum computing has been developing since the end of the $20^{\text {th }}$ century. A number of formal quantum computing models are constructed in which some computational problems, for example, [1-3], are solved more efficiently than in the classical computational model [4]. Actual information on the current level of quantum technologies development in the field of quantum computing is presented in $[5,6]$.

In [7] we have considered a method to implement $S$-boxes GOST R 34.12-2015 and AES by quantum circuits without using ancilla qubits, based on an algorithm for implementing an arbitrary unitary operator by means of a quantum circuit via decomposition of the corresponding unitary matrix into a product of two-level unitary matrices ([8, sec. 4.5]). Quantum circuits that implement $S$-boxes of GOST R 34.12-2015 and AES without ancilla qubits were constructed taking into account the optimization, based on the removal of sequences of quantum operations that are equivalent to the identical transformation.

For the implementation of GOST R 34.12-2015 "Magma" S-boxes 4 logical qubits are enough, and for the implementation of GOST R 34.12-2015 "Kuznechik" and AES $S$-boxes 8 logical qubit are sufficient, while in $[9,10]$ the implementation of AES $S$-box requires 40 logical qubits. In [10] a method for constructing a quantum circuit is described which implements the AES $S$-box on 9 logical qubits, but it is argued that in comparison with 40 logical qubits case, this implementation requires approximately three times more quantum gates.

In this paper to construct quantum circuits (Fig. 1-8) we use generalized $\operatorname{CNOT}(C \mid t)$ gates $([8,11])$, in which qubit $t$ is controlled by the set of qubits $C$. Generalized gates $\operatorname{CNOT}(C \mid t)$ may be implemented without using ancilla qubits ([8, p. 184]), therefore, we will consider generalized gates $\operatorname{CNOT}(C \mid t)$ as one self-independent logic gate, which is consistent with the techniques of assessing the quantum resources described in $[12,13]$.

\section{Construction of quantum circuits implementing $S$-boxes without ancilla qubits}

In this section, we present an algorithm for constructing quantum circuits that implement $S$-boxes without ancilla qubits, based on the decomposition of the substitution into independent cycles.

Table 1 presents the results of our implementation of Algorithm 1. 


\section{Algorithm 1}

Input: Substitution $s \in S\left(V_{n}\right)$.

Output: Quantum circuit for $s \in S\left(V_{n}\right)$ without ancilla qubits.

1: Represent $s$ as a product of independent cycles and remove fixed points. As a result, we obtain $k \in \mathbb{N}$ independent cycles, $s=s_{1} s_{2} \ldots s_{k}$.

2: Consider each cycle $s_{i}, i \in \overline{1, k}$ as an independent substitution, find the decomposition of the unitary matrix $U_{s_{i}}$ corresponding to the cycle $s_{i}, i \in \overline{1, k}$, into the product of two-level matrices (see $[7,8]$ ):

$$
U_{s_{i}}=V_{1}^{i} \cdot \ldots \cdot V_{t}^{i}
$$

3: By the matrices $V_{1}^{i}, \ldots, V_{t}^{i}$ we can determine all possible pairs of states

$$
\operatorname{data}_{i}=\left\{\left(x_{j}^{i}, y_{j}^{i}\right): V_{j}^{i}\left|x_{j}^{i}\right\rangle=\left|y_{j}^{i}\right\rangle, i \in \overline{1, k}, j \in \overline{1, t}, x_{j}^{i} \neq y_{j}^{i}\right\} .
$$

Lists data $_{i}$ may be simply written according to the cycles of $s_{i}, i \in \overline{1, k}$, by restoring the transition table of $s_{i}$. Description of formation of lists data $_{i}$ through two-level matrices is given in order to define $d a t a_{i}$ strictly and unambiguously.

4: For each pair $\left(x_{j}^{i}, y_{j}^{i}\right) \in \operatorname{data}_{i}, i \in \overline{1, k}, j \in \overline{1, t}$, define the list of bit numbers $\operatorname{numb}_{\left(x_{j}^{i}, y_{j}^{i}\right)} \subset\{1,2, \ldots, n\}$ containing $j$ iff $x_{j}^{i}$ is different from $y_{j}^{i}$.

5: Denote data $=\bigcup_{i=1}^{k} d a t a_{i}$. It is required to sort the elements of data in such a way that

$$
\sum_{w=1}^{\mid \text {data|-1 }}\left|\operatorname{numb}_{\left(x_{w}, y_{w}\right)} \cap \operatorname{numb}_{\left(x_{w+1}, y_{w+1}\right)}\right| \rightarrow \max ,
$$

moreover, the transitions $\left(x_{j}^{i}, y_{j}^{i}\right)$ obtained through the cycle $s_{i}$ must preserve the relative order (otherwise, instead of the cycle $s_{i}$ we will get some other cycle $s^{\prime}$ ). This stage may be implemented using a random search with restrictions. As a result of this sorting of data, we get data'.

6: To each element $\left(x^{\prime}, y^{\prime}\right) \in d a t a^{\prime}$ still corresponds some two-level matrix, i.e. each element of data' may be easily implemented using some simple quantum circuit (see $[7,8])$. The implementation of two-level matrices in the form of a quantum circuit may be ambiguous. Let $d_{\left(x^{\prime}, y^{\prime}\right)}$ be the Hamming distance between the binary representations $x^{\prime}$ and $y^{\prime}$, then there exists exactly $d_{\left(x^{\prime}, y^{\prime}\right)}$ ! various quantum circuits consisting of quantum gates CNOT and generalized $\mathrm{CNOT}(C \mid t)$ that implement the transition $\left(x^{\prime}, y^{\prime}\right)$ (see [8]). Among them there are only $d_{\left(x^{\prime}, y^{\prime}\right)}$ quantum circuits that differ significantly, which are determined by the number $t$ of the controlled qubit in quantum gates $\operatorname{CNOT}(C \mid t)$ that occurs during the implementation of two-level matrices. 
7: We assume that the quantum circuit that implements the transitions of data $^{\prime}$ may be optimized by independent parts. Then the search for an optimized quantum circuit for $s \in S\left(V_{n}\right)$ may be organized using the following procedure:

a: Initialize an array of $\left|d a t a^{\prime}\right|$ elements memory $=\{1, \ldots, 1\}$. In memory $[$ iter $]$ we will write the number of implementation of the two-level matrix that implements the transition $\left(x_{i t e r}^{\prime}, y_{i t e r}^{\prime}\right) \in d a t a^{\prime}$, iter $\in \overline{1,\left|d a t a^{\prime}\right|}$. Set iter $=1$.

b: Set the search depth, for example, depth $=3$. Until iter $<\mid$ data $^{\prime} \mid$ do:

i: Search for a quantum circuit with minimum length that implements transitions $\left(x_{i t e r}^{\prime}, y_{i t e r}^{\prime}\right), \ldots,\left(x_{i t e r+\operatorname{depth-1}}^{\prime}, y_{i t e r+\operatorname{depth-1}}^{\prime}\right)$ by iterating over all possible variants of quantum circuits that implements $\left(x_{i t e r}^{\prime}, y_{i t e r}^{\prime}\right), \ldots,\left(x_{i t e r+\operatorname{depth-1}}^{\prime}, y_{i t e r+\operatorname{depth-1}}^{\prime}\right)$. Write the obtained numbers of implementations of the two-level matrices to memory $[$ iter $], \ldots$, memory $[$ iter + depth -1$]$.

ii: iter $=$ iter + depth; The depth of the search is determined by the available computing power.

c: Repeat this procedure starting at iter $=2$ and the same value of depth.

8: As a result, we obtain a quantum circuit that implements $s \in S\left(V_{n}\right)$ without using ancilla qubits, with the minimal number of quantum gates.

Table 1: Data from algorithm 1 for implementing $S$-boxes without ancilla qubits

\begin{tabular}{|l|l|}
\hline$S$-box & data'-sequences of elementary qubit states transformations \\
\hline$\pi_{0}$ & $(14,15)(13,14)(11,14)(10,13)(9,14)(8,14)(7,9)(6,11)(12,0)(4,10)(3,6)(2,6)(1,4)$ \\
\hline$\pi_{1}$ & $(10,14)(11,12)(9,14)(7,12)(1,8)(6,10)(5,10)(4,9)(6,0)$ \\
\hline$\pi_{2}$ & $(1,3)(9,1)(13,9)(5,15)(7,13)(10,7)(6,10)(14,6)(2,5)(4,2)(8,14)(11,4)(0,11)$ \\
\hline$\pi_{3}$ & $(14,15)(13,14)(12,15)(11,13)(9,12)(8,15)(7,15)(6,15)(5,13)(4,13)(3,8)(1,8)(12,0)$ \\
\hline$\pi_{4}$ & $(5,1)(0,7)(2,5)(8,0)(14,2)(4,8)(13,4)(10,3)(11,14)(12,11)(15,12)$ \\
\hline$\pi_{5}$ & $(12,13)(11,13)(10,11)(9,10)(8,11)(7,10)(5,15)(6,12)(4,9)(2,15)(3,6)(5,0)(1,13)$ \\
\hline$\pi_{6}$ & $(13,15)(9,14)(12,13)(6,14)(5,9)(11,15)(10,11)(4,6)(3,5)(8,15)(1,14)(7,12)(8,0)$ \\
\hline$\pi_{7}$ & $(7,3)(1,7)(0,1)(4,0)(8,4)(6,8)(11,6)(14,11)(2,14)(15,2)(9,15)(12,9)(13,12)$ \\
\hline
\end{tabular}

In Fig. 1-8 the quantum circuits are presented that implement the $S$-boxes of GOST R 34.12-2015 "Magma". Optimization of quantum circuits that implement $S$-boxes GOST R 34.12-2015 "Magma" was carried out by "brute force", i.e. for "Magma" in Algorithm 1, we have set 
depth $=\left|d a t a^{\prime}\right|-1$. Comparison of the number of quantum gates in quantum circuits, at [7] and pic. 1-8, that implement the $S$-box without ancilla qubits is given in table 2 .

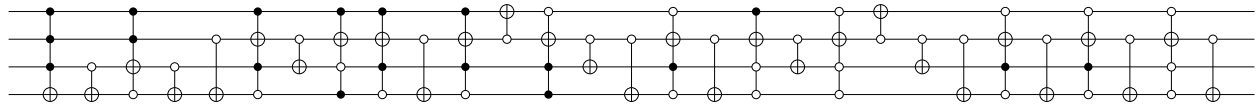

Figure 1: Quantum circuit for $\pi_{0}=(12,4,6,2,10,5,11,9,14,8,13,7,0,3,15,1)$

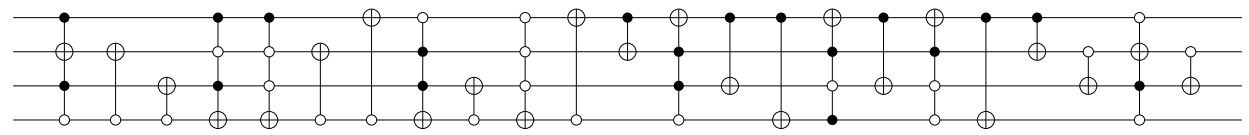

Figure 2: Quantum circuit for $\pi_{1}=(6,8,2,3,9,10,5,12,1,14,4,7,11,13,0,15)$

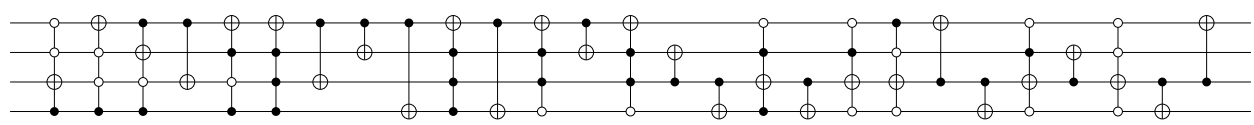

Figure 3: Quantum circuit for $\pi_{2}=(11,3,5,8,2,15,10,13,14,1,7,4,12,9,6,0)$

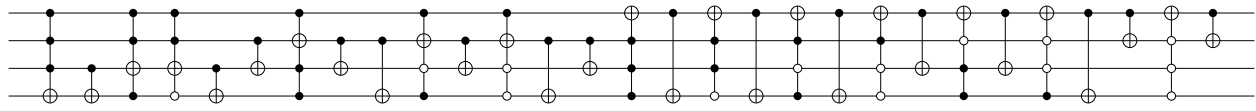

Figure 4: Quantum circuit for $\pi_{3}=(12,8,2,1,13,4,15,6,7,0,10,5,3,14,9,11)$

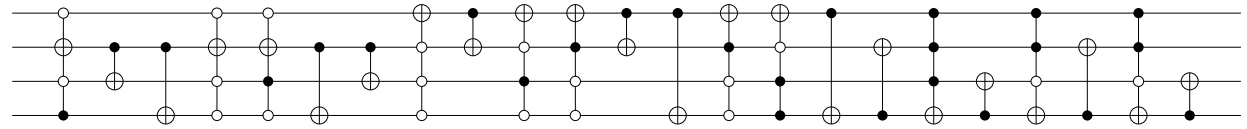

Figure 5: Quantum circuit for $\pi_{4}=(7,15,5,10,8,1,6,13,0,9,3,14,11,4,2,12)$

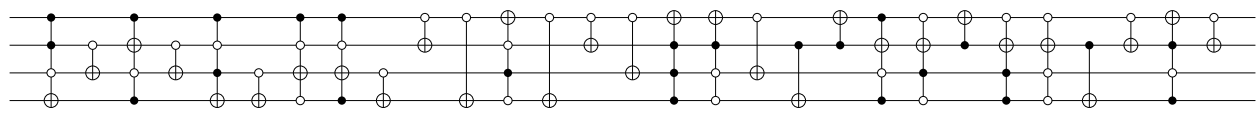

Figure 6: Quantum circuit for $\pi_{5}=(5,13,15,6,9,2,12,10,11,7,8,1,4,3,14,0)$ 


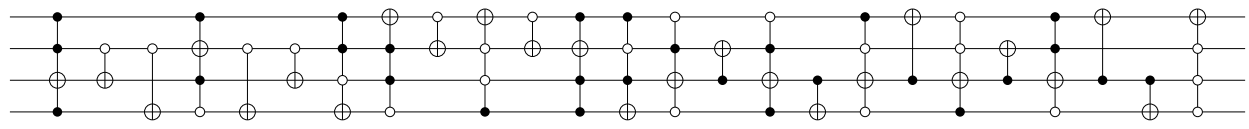

Figure 7: Quantum circuit for $\pi_{6}=(8,14,2,5,6,9,1,12,15,4,11,0,13,10,3,7)$

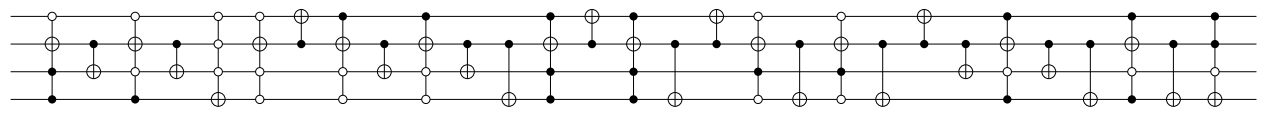

Figure 8: Quantum circuit for $\pi_{7}=(1,7,14,13,0,5,8,3,4,15,10,6,9,12,11,2)$

Source code for verifying the correctness of quantum circuits in the quantum simulator Quipper [14], example for $\pi_{4}$.

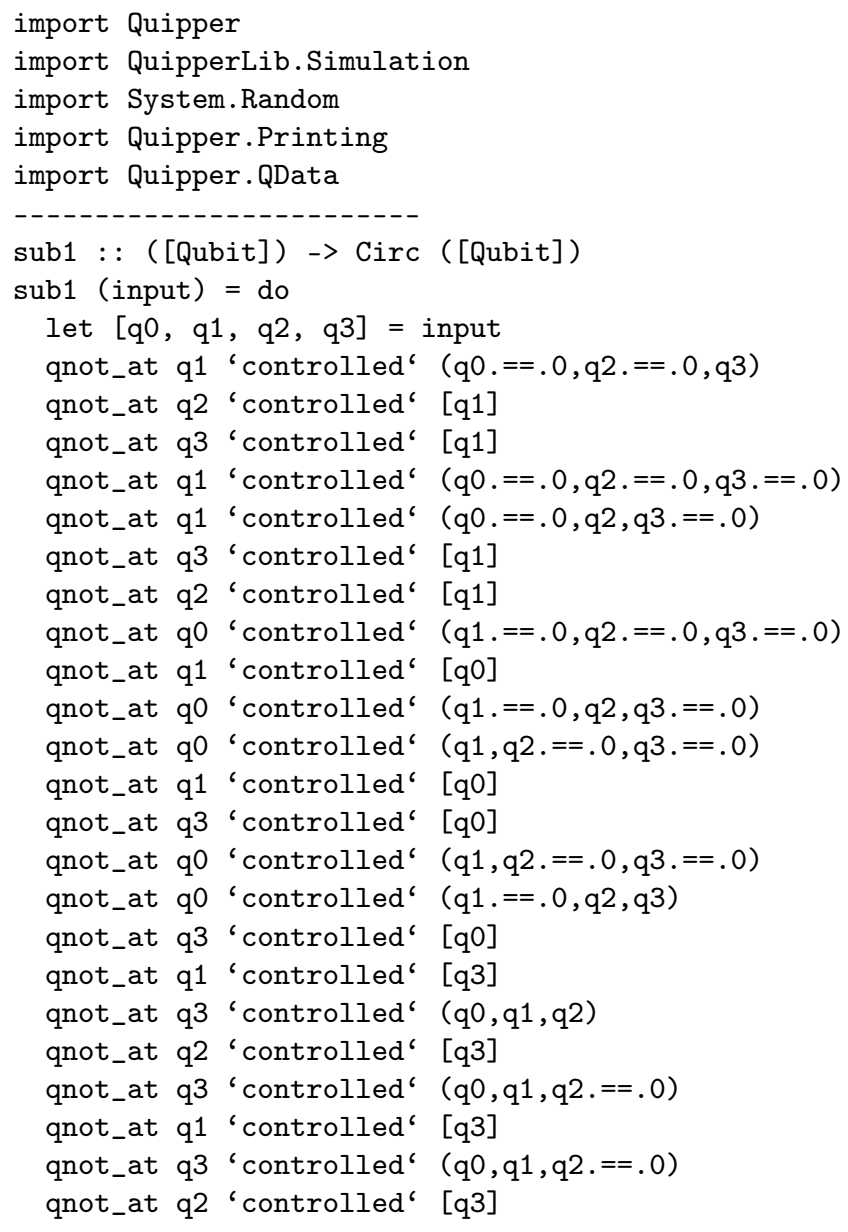


$\operatorname{return}([q 0, q 1, q 2, q 3])$

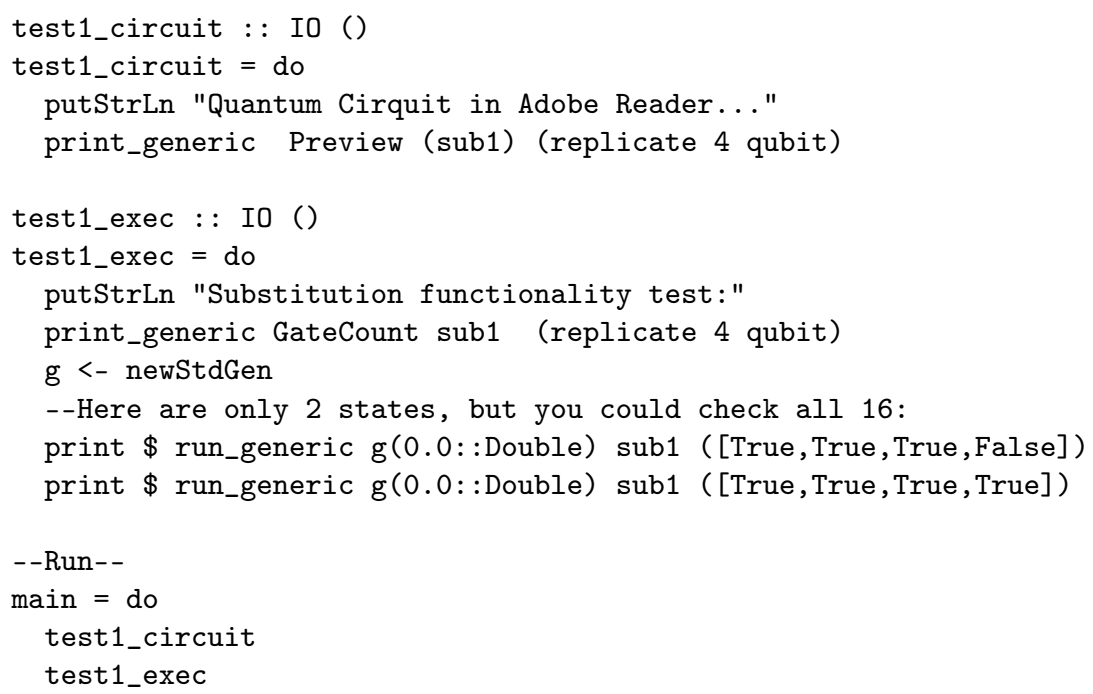

Table 2: Comparison of the number of quantum gates in quantum circuits, at [7] and Fig. 1-8, that implement the $S$-box without ancilla qubits

\begin{tabular}{|c|c|c|c|}
\hline$S$-box & $\begin{array}{c}\text { Number of } \\
\text { cycles in } \\
S \text {-box }\end{array}$ & $\begin{array}{c}\text { Total gates in } \\
\text { quantum circuit [7] }\end{array}$ & $\begin{array}{c}\text { Total gates in new } \\
\text { quantum circuit }\end{array}$ \\
\hline$\pi_{0}$ & 2 & 33 & 29 \\
\hline$\pi_{1}$ & 3 & 29 & 23 \\
\hline$\pi_{2}$ & 2 & 37 & 27 \\
\hline$\pi_{3}$ & 1 & 29 & 29 \\
\hline$\pi_{4}$ & 3 & 31 & 29 \\
\hline$\pi_{5}$ & 2 & 35 & 25 \\
\hline$\pi_{6}$ & 2 & 31 & 29 \\
\hline$\pi_{7}$ & 1 & 31 & 23 \\
\hline
\end{tabular}

\section{Conclusion}

We have obtained new quantum circuits implementing GOST R 34.122015 "Magma" $S$-boxes on 4 logical qubits with fewer logical quantum gates than in [7]. The obtained results allow to suppose that the more cycles in 
substitution, the less the length of the quantum circuit implementing this substitution may be.

\section{References}

[1] Shor P.W., "Polynomial-time algorithms for prime factorization and discrete logarithms on a quantum computer", SIAM J. Comput., 26:5 (1997), 1484-1509.

[2] Grover L.K., "A fast quantum mechanical algorithm for database search", Proc. 28th Annu. ACM Symp. Theory of Computing (STOC), 1996, 212-219.

[3] Simon D.R., "On the power of quantum computation", SIAM J. Comput., 26:5 (1997), 1474-1483.

[4] Gainutdinova A.F., Comparative complexity of quantum and classical models of calculations., Thesis for the degree of Candidate of Phys.-Math. Sci., 2004.

[5] "Quantum Computing Report", https://quantumcomputingreport.com.

[6] Quantum Computing: Progress and Prospects, Nat. Acad. Sci., Engin., and Medicine, The Nat. Acad. Press, Washington, DC, 2018, 202 pp.

[7] Denisenko D.V., "Quantum circuits for substitution implementation without ancilla qubits", Zh. Exper. Teor. Fis., 155:6 (2019), 999-1008 (in Russian).

[8] Nielsen M.A., Chuang I.L., Quantum computation and quantum information, Cambridge Univ. Press, 2010, xxxii +676 pp.

[9] Kim P., Han D., Jeong K.C., "Time-space complexity of quantum search algorithms in symmetric cryptanalysis: applying to AES and SHA-2", Quantum Inf. Process., 17:339 (2018).

[10] Grassl M., Langenberg B., Roetteler M., Steinwandt R., Applying Grover's algorithm to AES: quantum resourse estimation, Cryptology ePrint Archive, Report 2019/103, 2015.

[11] Younes A., Miller J., Automated method for building CNOT based quantum circuits for Boolean functions, Cryptology ePrint Archive, Report quant-ph/0304099v1, 2003.

[12] Jaques S., Schanck J. M., Quantum cryptanalysis in the RAM model: Claw-finding attacks on SIKE, Cryptology ePrint Archive, Report 2019/103, 2019.

[13] Submission requirements and evaluation criteria for the post-quantum cryptography standardization process, National Institute of Standards and Technology, 2017.

[14] The Quipper Language, http://www.mathstat.dal.ca/ selinger/quipper/. 


\section{Appendix 1}

The algorithm for constructing quantum circuits of an arbitrary unitary operator is described in [8], Section 4.5.

Definition. Let $N=2^{n}, n \in \mathbb{N}$, and $e_{1}, e_{2}, \ldots, e_{N}$ be the basis of the vector space $L_{\mathbb{C}^{N}}$ over the field of complex numbers $\mathbb{C}$. The unitary matrices $U \in$ $\mathbb{C}_{2^{n}, 2^{n}}$, nontrivially acting on no more than two basis vectors $e_{1}, e_{2}, \ldots, e_{N}$, are called two-level unitary matrices (see [8], section 4.5.1).

Let's construct a quantum circuit that implements

$$
\pi_{1}=(6,8,2,3,9,10,5,12,1,14,4,7,11,13,0,15) .
$$

The substitution $\pi_{1} \in S\left(V_{4}\right)$. Denote $y=\pi_{1}(x), x, y \in V_{4}$. The states $|x\rangle,|y\rangle$ are vector-columns from $L_{\mathbb{C}^{4}}$, the action of the operator $U|x\rangle=|y\rangle$ is a multiplication of the column vector $|x\rangle$ by the matrix $U \in \mathbb{C}_{2^{4}, 2^{4}}$.

1. The unitary matrix for $\pi_{1}$ :

$$
U_{\pi_{1}}=\left(\begin{array}{cccccccccccccccc}
0 & 0 & 0 & 0 & 0 & 0 & 0 & 0 & 0 & 0 & 0 & 0 & 0 & 0 & 1 & 0 \\
0 & 0 & 0 & 0 & 0 & 0 & 0 & 0 & 1 & 0 & 0 & 0 & 0 & 0 & 0 & 0 \\
0 & 0 & 1 & 0 & 0 & 0 & 0 & 0 & 0 & 0 & 0 & 0 & 0 & 0 & 0 & 0 \\
0 & 0 & 0 & 1 & 0 & 0 & 0 & 0 & 0 & 0 & 0 & 0 & 0 & 0 & 0 & 0 \\
0 & 0 & 0 & 0 & 0 & 0 & 0 & 0 & 0 & 0 & 1 & 0 & 0 & 0 & 0 & 0 \\
0 & 0 & 0 & 0 & 0 & 0 & 1 & 0 & 0 & 0 & 0 & 0 & 0 & 0 & 0 & 0 \\
1 & 0 & 0 & 0 & 0 & 0 & 0 & 0 & 0 & 0 & 0 & 0 & 0 & 0 & 0 & 0 \\
0 & 0 & 0 & 0 & 0 & 0 & 0 & 0 & 0 & 0 & 0 & 1 & 0 & 0 & 0 & 0 \\
0 & 1 & 0 & 0 & 0 & 0 & 0 & 0 & 0 & 0 & 0 & 0 & 0 & 0 & 0 & 0 \\
0 & 0 & 0 & 0 & 1 & 0 & 0 & 0 & 0 & 0 & 0 & 0 & 0 & 0 & 0 & 0 \\
0 & 0 & 0 & 0 & 0 & 1 & 0 & 0 & 0 & 0 & 0 & 0 & 0 & 0 & 0 & 0 \\
0 & 0 & 0 & 0 & 0 & 0 & 0 & 0 & 0 & 0 & 0 & 0 & 1 & 0 & 0 & 0 \\
0 & 0 & 0 & 0 & 0 & 0 & 0 & 1 & 0 & 0 & 0 & 0 & 0 & 0 & 0 & 0 \\
0 & 0 & 0 & 0 & 0 & 0 & 0 & 0 & 0 & 0 & 0 & 0 & 0 & 1 & 0 & 0 \\
0 & 0 & 0 & 0 & 0 & 0 & 0 & 0 & 0 & 1 & 0 & 0 & 0 & 0 & 0 & 0 \\
0 & 0 & 0 & 0 & 0 & 0 & 0 & 0 & 0 & 0 & 0 & 0 & 0 & 0 & 0 & 1
\end{array}\right) .
$$

2. The matrix $U_{\pi_{1}}$ may be represented as a product of two-level unitary matrices:

$$
U_{\pi_{1}}=V_{1} \cdot V_{2} \cdot V_{3} \cdot V_{4} \cdot V_{5} \cdot V_{6} \cdot V_{7} \cdot V_{8} \cdot V_{9} .
$$

The table 3 contains two-level matrices $V_{1}, \ldots, V_{9}$, participating in the decomposition $U_{\pi_{1}}$, states $s$ and $t$, on which two-level matrices act nontrivially, and quantum circuits implementing two-level matrices $V_{1}, \ldots, V_{9}$. Matrices are written as a list of strings, each row is a vector $v_{i} \in V_{16}$, $\left\|v_{i}\right\|=1, i \in \overline{1,16}$ and written in hexadecimal notation. 
Table 3: Representation of the matrix $U_{\pi_{1}}$ as a product of two-level matrices $U_{\pi_{1}}=V_{1} \cdot V_{2} \cdot V_{3} \cdot V_{4} \cdot V_{5} \cdot V_{6} \cdot V_{7} \cdot V_{8} \cdot V_{9}$

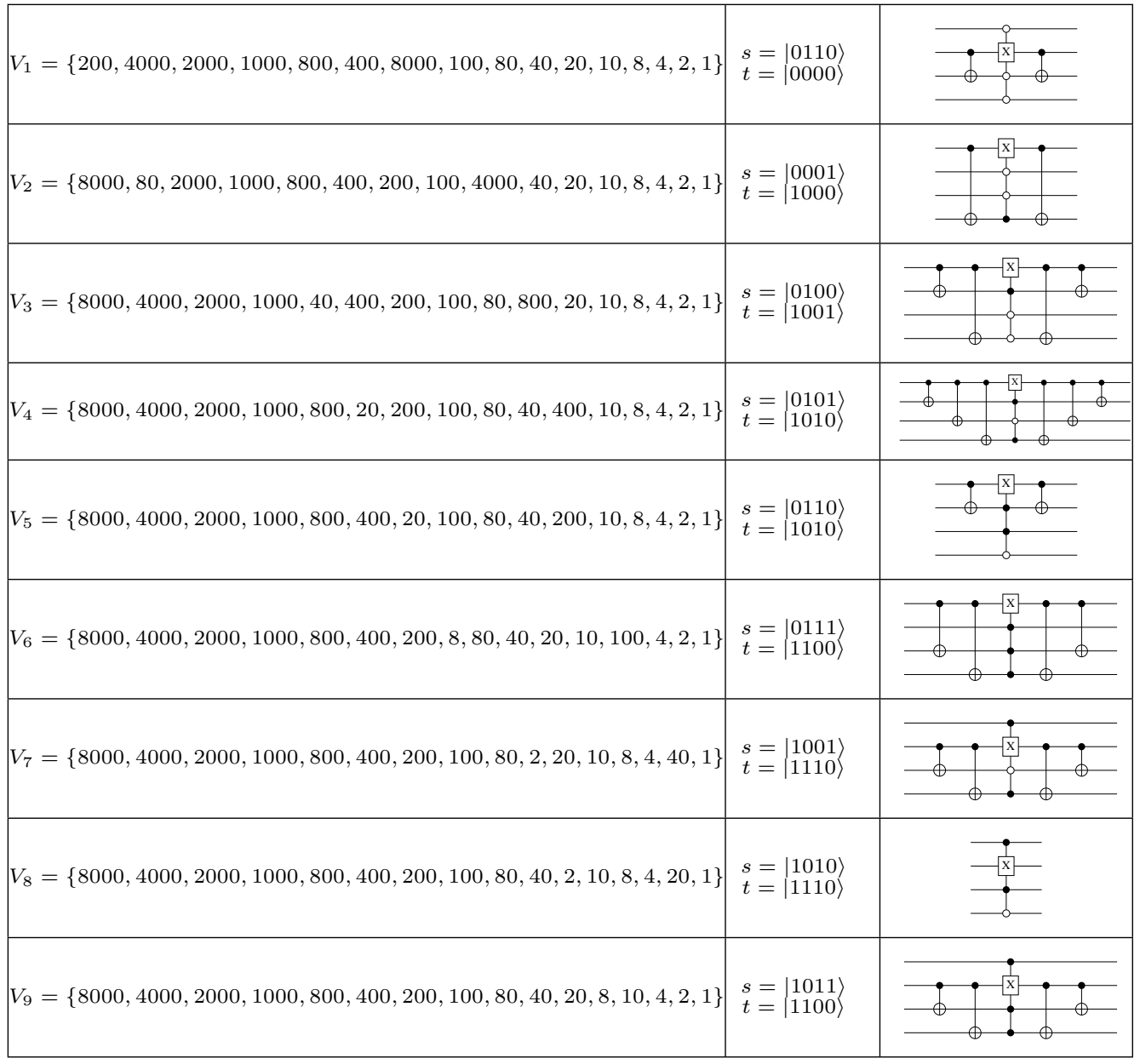

Since $|y\rangle=U|x\rangle,|y\rangle=V_{1} \cdot\left(\ldots \cdot\left(V_{8} \cdot\left(V_{9} \cdot|x\rangle\right)\right) \ldots\right)$, the quantum circuit of $U_{\pi_{1}}$ is as follows:

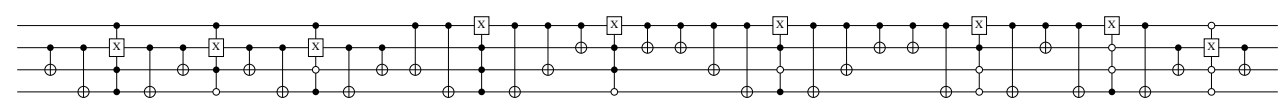

Figure 9: Quantum circuit $\pi_{1}=(6,8,2,3,9,10,5,12,1,14,4,7,11,13,0,15)$

After optimization of the quantum circuit in the Fig. 9, we obtain the quantum circuit in the Fig. 2. 